\title{
Low Energy Cosmic Rays Emitted by the Orion Complex into the Local Medium
}

\author{
V. Schönfelder ${ }^{1}$, V.A. Dogiel ${ }^{2}$, M.J. Freyberg ${ }^{1}$, and G.E. Morfill ${ }^{1}$ \\ 1 Max-Planck-Institut für extraterrestrische Physik, D-85740 Garching, Germany \\ ${ }^{2}$ P.N.Lebedev Physical Institute, 117924 Moscow, Russia
}

\begin{abstract}
The excess of $\gamma$-ray emission in the energy range $3-7 \mathrm{MeV}$ discovered by COMPTEL in the direction of the Orion complex may imply that there is a powerful source of low energy cosmic rays in the local galactic medium. Several interpretations of the excess have been suggested. One of them assumes that the emission is identified with nuclear de-excitation lines of excited ${ }^{16} \mathrm{O}$ and ${ }^{14} \mathrm{C}$. To provide the excess the energy deposit in these nuclei should be as large as $\sim 10^{39}$ erg $\mathrm{s}^{-1}$. If a comparable part of these nuclei escape into the local galactic medium they can play a significant role in the energy balance there.

If the excess is interpreted as continuous it can be produced by bremsstrahlung emission of fast $10 \mathrm{MeV}$ electrons. Much less energy deposit in these electrons is necessary $\left(\sim 10^{36} \mathrm{erg} \mathrm{s}^{-1}\right)$ to generate the observed Orion flux. The intensity of these electrons even near Earth can be higher than that of the galactic cosmic ray electrons.
\end{abstract}

\section{Introduction}

One of the key problems of the local galactic medium is the question whether the conditions there are the same as in the other parts of the interstellar medium or whether this part of the Galaxy heated by several supernova explosions has rather peculiar parameters. This question is especially interesting in the context of the origin of cosmic rays since we do not know (for sure) whether the characteristics of cosmic rays observed near Earth differ (or not) from those in other parts of the Galaxy.

On the other hand one of the key assumptions of cosmic ray physics is that the parameters of galactic cosmic rays are not changed significantly in the local galactic medium. Only then it is possible to derive the cosmic ray lifetime in the Galaxy from the chemical composition of cosmic rays measured near Earth.

Radio and $\gamma$-ray investigations seem to confirm this assumption since analyses of radio- and $\gamma$-ray background radiations do not show significant spatial variations of the spectrum and the density of galactic cosmic rays in comparison with that observed near Earth. On the other hand, $\gamma$-ray data may discover a near-by powerful source of cosmic rays with a luminosity as high as about $3 \cdot 10^{38} \mathrm{erg} \mathrm{s}^{-1}$ which may have a strong influence on the conditions in the local interstellar space. Below we discuss briefly this possibility. 


\section{Flux of Gamma-Rays from Orion}

COMPTEL has detected strong 3 to $7 \mathrm{MeV} \gamma$-ray emission from the Orion complex with no significant evidence for emission outside this range. Its spectrum shows features that might be due to splitting of $\mathrm{C}$ and $\mathrm{O}$ nuclear interaction lines (Bloemen et al., 1997) The detected excess from Orion in the energy range $3-7 \mathrm{MeV}$ has a value of

$$
F_{\gamma} \sim(10.1 \pm 1.4) \times 10^{-5} \mathrm{ph} \mathrm{cm}^{-2} \mathrm{~s}^{-1}
$$

To produce the observed excess in the energy range $3-7 \mathrm{MeV}$ in the direction of Orion the photon flux should be about

$$
L_{\gamma} \sim 3 \cdot 10^{39} \mathrm{ph} \mathrm{s}^{-1}
$$

or the luminosity in the $4.4 \mathrm{MeV}$ line should be

$$
F_{\gamma} \sim E_{\gamma} L_{\gamma} \sim 2 \cdot 10^{34} \mathrm{erg} \mathrm{s}^{-1} .
$$

To satisfy the observational data obtained by COMPTEL and EGRET we should assume that the spectrum of accelerated particles is truncated at an energy $E_{m}$. Its value is less than $100 \mathrm{MeV} / \mathrm{n}$ and higher than $20-30 \mathrm{MeV} / \mathrm{n}$.

In the framework of the thick target model the total luminosity of cosmic ray nuclei $L_{n}$ needed to produce the Orion $\gamma$-ray flux (in the energy range of nuclei $E_{k}<E_{m}$ ) is estimated as (see Bykov and Bloemen, 1994)

$$
L_{n} \sim 10^{42} \mathrm{n} \mathrm{s}^{-1}
$$

or the energy luminosity of the Orion complex in these nuclei is

$$
F_{n} \sim 8 \cdot 10^{38} \mathrm{erg} \mathrm{s}^{-1} .
$$

\section{Orion Cosmic Ray Nuclei in the Local Medium}

The estimate (3) was derived under the assumption that the nuclei lose their energy by ionization only. On the other hand one cannot exclude that a part of the nuclei escape from Orion into the local galactic medium and reach the solar system. In this case their density near Earth is determined from the diffusion equation describing cosmic ray propagation in the Galaxy:

$$
N_{n}=\frac{L_{n}}{4 \pi r D}
$$

where $r$ is the distance between Orion and the Sun, $D$ is the diffusion coefficient in the local galactic medium, and $L_{n}$ is the production rate of nuclei in Orion.

From Eq.(4) we can easily estimate the flux of local nuclei if they are emitted by Orion, since we know almost all necessary parameters. The distance 
$r \sim 500 \mathrm{pc}$, the local diffusion coefficient $D$ was determined by Dorman et al. (1984), from the anisotropy of very high energy cosmic rays $\left(E \sim 10^{12}-10^{13}\right.$ eV) near Earth: $D \simeq 10^{29} \mathrm{~cm}^{2} \mathrm{~s}^{-1}$. Energy variations of the diffusion coefficient were derived from the cosmic ray chemical composition. It was concluded that its value is approximately constant at energies $E<1 \mathrm{GeV}$ and rises as $D \propto E^{0.6}$ at $E>1 \mathrm{GeV}$ (see e.g. Berezinsky et al. 1990). Thus, extrapolating the diffusion coefficient value into the energy range of $\leq 1 \mathrm{GeV}$ we obtain $D \sim 10^{27}-10^{28} \mathrm{~cm}^{2} \mathrm{~s}^{-1}$. The average galactic diffusion coefficient was derived from analyses of the galactic non-thermal emission and the cosmic ray chemical composition (Berezinskii et al. 1990, Strong et al. 1997). Its value may be as large as $10^{29} \mathrm{~cm}^{2} \mathrm{~s}^{-1}$ at energies $E \leq 1 \mathrm{GeV}$.

As we mentioned already the estimate (3) was derived under the assumption that the nuclei do not escape from Orion. If, however, a comparable flux of nuclei escapes into the local galactic medium, this results in an energy density of nuclei from Orion near Earth as high as $0.1-0.3 \mathrm{eV} \mathrm{cm}^{-3}$ even for a diffusion coefficient of $3 \cdot 10^{28} \mathrm{~cm}^{2} \mathrm{~s}^{-1}$. This energy density is comparable to that of galactic cosmic rays. Since the energy of cosmic rays in Orion is rather low they can play a significant role in the energy and ionization balances in the local medium.

\section{$410 \mathrm{MeV}$ Electrons in the Local Medium from Orion}

If the 3 to $7 \mathrm{MeV}$ excess is interpreted as continuous emission, it can be produced by bremsstrahlung emission of fast electrons, which have an almost energy independent spectrum below $20 \mathrm{MeV}$ and a cutoff at about $20 \mathrm{MeV}$. The energy needed to produce the Orion $\gamma$-ray excess is much less than in the case of the line origin. Only $10^{36} \mathrm{erg} \mathrm{s}^{-1}$ is necessary to produce the observed Orion $\gamma$-ray flux. If part of these electrons escape into the local interstellar medium, their density there may be significant and exceed that of the galactic cosmic ray electrons.

Direct observations of the local spectrum of cosmic ray electrons are restricted to energies above $1 \mathrm{GeV}$ because of the modulation in interplanetary space, but contrary to the nuclear component the electron component can be derived at low energies from observations of the Galactic non-thermal radio and $\gamma$-ray emission.

Especially, the electron spectrum derived from the diffuse galactic $\gamma$-ray emission, measured by COMPTEL, was extended down to the energy of 1 $\mathrm{MeV}$ (Strong et al. 1997). This analysis has been performed in the framework of the diffusion model of cosmic ray propagation in the Galaxy. It was shown that the intensity of the Galactic electrons at energies of $10 \mathrm{MeV}$ is about $2 \cdot 10^{-1} \mathrm{el} \mathrm{cm}^{-2} \mathrm{~s}^{-1} \mathrm{sr}^{-1}$.

A lower limit to the $10 \mathrm{MeV}$ electron production by Orion can be derived in the framework of the thick target model from the Orion $\gamma$-ray excess, $L_{e} \sim 3 \cdot 10^{39} \mathrm{el} \mathrm{s}^{-1}$. If a comparable flux of electrons escapes into the local 
galactic medium, this results in an intensity of electrons near Earth from Orion (using Eq.(4)) of about 1 to $10^{-2} \mathrm{el} \mathrm{cm}^{-2} \mathrm{~s}^{-1} \mathrm{sr}^{-1}$ depending on the value of the diffusion coefficient in the local Galactic space. In this respect the Orion source of $10 \mathrm{MeV}$ electrons may play an essential role in the energy balance of the local galactic medium.

Acknowledgements. VAD is grateful to his colleagues from the Max-PlanckInstitut für extraterrestrische Physik (Garching) for helpful and fruitful discussions. This work was partly supported by the Government of Russia through the Russian Foundation of Fundamental Researches under the grant No. 95-02-06422-a.

\section{References}

Berezinsky V.S., Bulanov S.V., Dogiel V.A., Ginzburg V.L., Ptuskin V.S., 1990, Astrophysics of Cosmic Rays, (ed. V.L.Ginzburg), North Holland

Bloemen H., Bykov A.M., Bozhokin S.V., Diehl R., Hermsen W., van der Meulen R.D., Oberlack U., Ryan J., Schönfelder V., Strong, A.W., Winkler C., 1997, ApJ, 475, L25

Bykov A.M., Bloemen H., 1994, A\&A 283, L1

Dorman L.I., Ghosh A., Ptuskin V.S., 1984, Sov.Astron.Lett. 10, 345

Strong A.S., Bennett K., Diehl R., Hermsen W., Purcell W., Schönfelder V., Stacy J.G., Winkler C., Youssefi G., 1997, A\&A Suppl. 120, 381 